courses are more or less comparable to North American university courses for physics students, and possibly at a level comparable to that of the UK introductory course described in my paper?

H. Neumann: As I'm not familiar with the US system, I rather would like to compare the German 13th (perhaps even 12th) grade to introductory college levels. Comparison must be checked from the details, as for the UK system. But the similarity with the UK system might be higher.

\title{
TEACHING ASTRONOMY AT KEIO SENIOR HIGH SCHOOL,
} JAPAN

Yukimasa Tsubota

Keio Senior High School, 4-1-2 Hiyoshi, Kouhoku-ku, Yokohama-shi 223, Japan

\section{Introduction}

The major problem in teaching astronomy in our senior high schools has to do with the nature of the Japanese educational system. The typical science curriculum consists of physics, chemistry, biology, earth science, and general science I \& II. The Japanese Ministry of Education allows General Science I to fulfill the minimum high-school graduation requirement in science. General Science I covers the basics of earth science. Astronomy has been taught as a part of General Science I and Earth Science.

Table 1. The Japanese Ministry of Education's Current Educational Guideline

\begin{tabular}{llcc}
\hline Subject Area & Subjects & Credits $^{a}$ & Statistics $^{b}$ \\
\hline \multirow{4}{*}{ Science } & Gen. Science I & 4 & Required Course \\
& Gen. Science II & 2 & Uncommon \\
& Physics & 4 & $22 \%$ \\
& Chemistry & 4 & $39 \%$ \\
& Biology & 4 & $32 \%$ \\
& Earth Science & 4 & $7 \%$ \\
\hline
\end{tabular}

${ }^{a}$ One credit $=35$ classroom hours of lessons. One classroom hour $=50$ minutes. From text sales. 
Many Japanese high schools do not offer earth science because it is not covered in the college entrance exams (Table 1). Moreover, teachers usually spend many hours with the students memorizing the basics of earth science rather than allowing some time in the laboratory.

Keio Senior High School has a unique and creative academic curriculum (Table 2) that is not influenced by the Japanese college entrance exam because the graduates are admitted to Keio University without the entrance exam. The school was founded in 1886, has 130 faculty members, and 2567 students (all boys) in grades 10 to 12 . It is located on the campus of Keio University, 20 minutes by train from Yokohama, and 30 minutes by fast train from Tokyo.

\section{Lesson Plans}

Keio's tenth-grade earth-science schema are as follows. The teaching materials cover the Earth, sun, solar system, and stellar evolution. The objectives of these plans are: 1) to have students understand nature via experiments and exercises, and 2) to have them learn scientific methodology and to acquire deductive reasoning through these materials. The meaning of the different kinds of brackets, and of the use of italics, is given at the very end of this section.

Table 2. Keio's Science Curriculum

\begin{tabular}{lccc}
\hline Subjects & Credits & Grades & Statistics (1988) \\
\hline Biology & 4 & 10 & Required \\
Earth Science & 3 & 10 & Required \\
Chemistry & 3 & 11 & Required \\
Physics & 3 & 11 & Required \\
Advanced Chemistry & 3 & 12 & $196(23 \%)$ \\
Advanced Physics & 3 & 12 & $196(23 \%)$ \\
Projects on Physics & 2 & 12 & $288(33 \%)$ \\
Projects on Earth Science & 2 & 12 & $75(9 \%)$ \\
\hline
\end{tabular}

Lesson Plan 1: The Earth (22 lessons)

Earth dimension (3)

- Earth's shape: evidence

- [Earth ellipsoid]: Eratosthenes' method, oblateness, curvature

The motion of the Earth (16)

- [Positional astronomy]: horizontal coordinates and equatorial coordinates, meridian, zenith 
- $\{$ Solar time and sidereal time\}: definition of time, local time, universal time, time difference, equation of time

- Apparent motions of celestial objects: diurnal motion, altitude of meridian transit, circumpolar star

- [Foucault's pendulum]: rotation of the Earth, coriolis effect

- [Apparent motion of sun and planets]: direct motion, retrograde motion, stationary, from geocentric theory to heliocentric theory

- Revolution of the Earth: annual aberration, annual parallax, distance of fixed stars

- $\{$ Synodic period and sidereal period\}: conjunctions: inferior conjunction, superior conjunction, greatest elongation, opposition

- [Orbit of Mars]: ecliptic coordinates, Kepler's laws

- Moon's motion: law of universal gravitation, circular motion, \{altitude of artificial satellites\}

Our solar system (2)

- Comparative planetology: radius, mass, density, materials, gravity, rotation period, sidereal period, atmosphere

- $\{$ Length of the day\}: effect of sidereal motion.

- <Planetary exploration>

Recognizing the peculiarity of the Earth (1)

Lesson Plan 2: The Sun (23 lessons)

The surface of the sun (9)

- [Sunspot and facula]; <the sun>; revolution period of sun, sunspot distribution, 11-year period of sunspot and climate change, (introdution to statistical analysis)

Energy source of the sun (5)

- [Direct solar radiation]: solar constant \& atmospheric transmissivity

- Speculation on solar energy source: comparison of energy sources, chemical energy, gravitational energy, atomic energy

- $\{$ Determination of the solar surface temperature\}; Stefan-Boltzmann law, albedo, effective temperature, and greenhouse effect

Composition of the sun (4)

- [Spectroscopic observation of the sun]: continuous spectrum, emission spectrum, absorption spectrum, Fraunhofer lines, <solar activity > 
- [Spectroscopic astronomy]: spectral types, stellar surface temperature, Wien's law, determination of solar surface temperature

The sun as a star (4)

- \{Absolute magnitude and visual magnitude\}; luminosity, magnitude, distance

- [Hertzsprung-Russell diagram]: classification of stars, main-sequence stars, giant stars, supergiant stars, white dwarfs, spectroscopic parallax

- Stellar evolution: solar evolution

Introduction to modern astronomy $(1)$ : <x-ray astronomy $>$

( ) school hours

[ ] laboratory works

\{\} calculation exercise

$<>$ audio-visual material

discussion lab

\section{Discussion}

I believe that astronomy is a suitable subject to teach scientific methodology and to introduce deductive reasoning. However, some students have been disappointed in our methods of teaching astronomy. Perhaps it is the result of the discrepancy between the material we teach and the students' interest, which run more to constellations and astrology. Students do not seem especially interested in astronomy as a laboratory science. Learning astronomy can be very difficult without the fundamentals of physics and advanced mathematics.

So is astronomy needed at a senior high school? My answer is "yes." Conceptual astronomy should be developed for senior-high school students. Some laboratory work might be delayed until the college level. 